
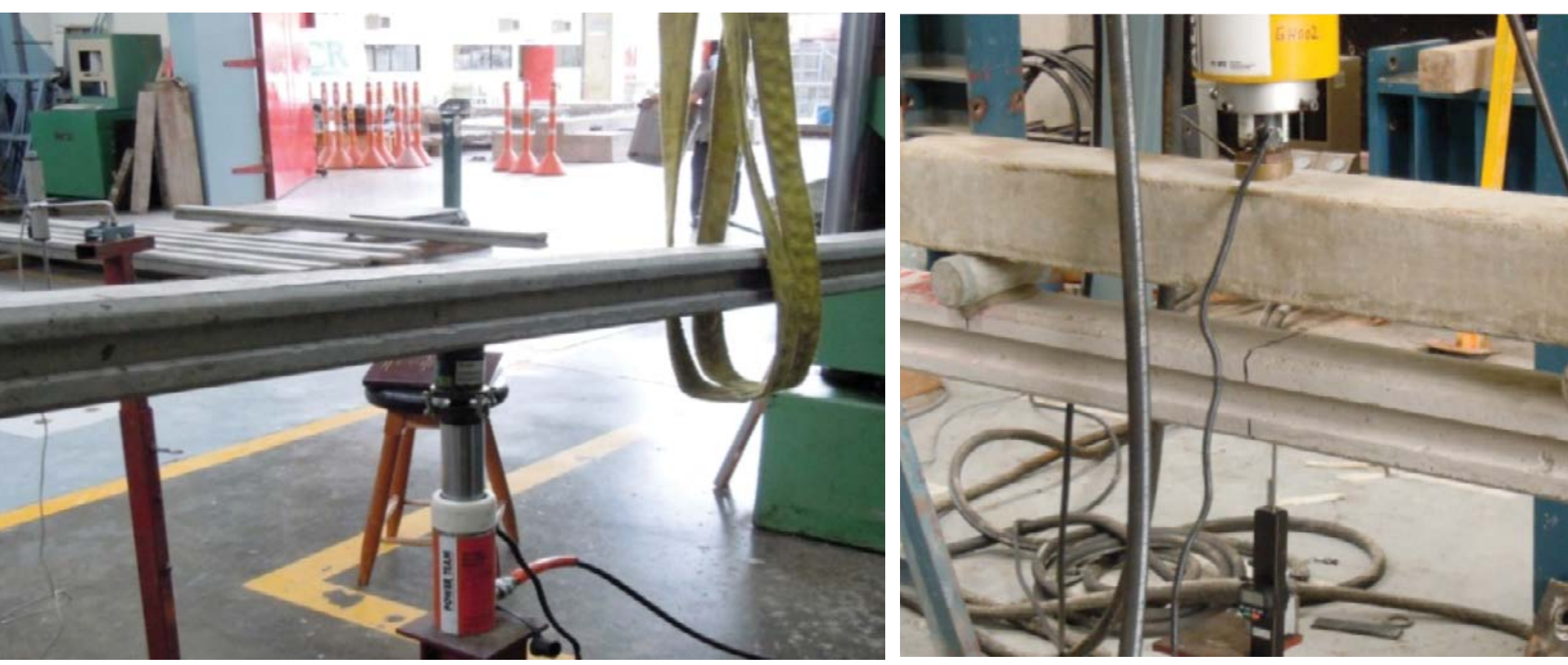

\title{
Pruebas experimentales para determinar la capacidad de columnas de concreto prefabricado
}

Fecha de recepción: 18 de junio del 2012

Fecha de aceptación: 30 de julio del 2012

\section{Resumen}

Este documento desarrolla un método para comparación y correlación de dos métodos para prueba de columnas prefabricadas de concreto reforzado. El objetivo principal de este artículo es simplificar el control de calidad en este campo y brindar información para extrapolar los resultados que se obtengan de un método de prueba más sencillo como el ASTM C78M, Método Estándar para la Capacidad en flexión del Concreto (usando una viga simple cargada en el tercio medio) a un método con un modelo más cercado al modelo analítico comúnmente utilizado para determinar el estado de fuerzas internas de la columna, como el Método para la Capacidad en flexión del Concreto (usando una viga empotrada en 0,220 $\cdot$ L y cargada a los 0,728 · L.). Para la investigación fueron evaluadas 5 fábricas productoras de columnas, Para cada fábrica, fueron fallas 7 columnas con un método y 7 columnas con el otro método. El resultado experimental indica que aparentemente no existe diferencia significativa entre los dos métodos.

\section{Palabras claves:}

Ing. Francisco Villalobos Ramírez, francisco.villalobos@ucr.ac.cr Ing. José Pablo Alfaro Martínez, josepablo.alfaro@ucr.ac.cr LanammeUCR

\section{Abstract}

This document develops a method for comparison and correlation of two methods for testing reinforced concrete prefabricated columns. The main objective of this article is to simplify the quality control methods in this field and bring information on how to extrapolate the results that may be obtained from a simpler testing method as the ASTM C78M, Standard Test Method for Flexural Strength of Concrete (using simple beam with third-point loading) to a method which represents better the condition of loading that often are used in analytical design of this elements, which is the Method for Flexural Strength of Concrete (using cantilevered beam at $0,220 \cdot L$ and loaded at $0,728 \cdot L)$. For the investigation 5 producing factories of columns were evaluated, For each factory, were 7 columns were tested with a method and 7 columns with the other method. The experimental result indicates that apparently does not exist significant difference between both methods.

\section{Keywords:}

Prefabricated columns, third-point loading, cantilever loading, flexural strength. 


\section{Agradecimientos}

Se desea extender un agradecimiento a las siguientes empresas productoras de elementos prefabricados por donar sus productos para cumplir con el objetivo del presente estudio:

Eurobau Costa Rica Productos de Concreto Prefabricado. Ekstrom Costa Rica.

IDECASA.

INPREFA.

Pretensados Nacionales.

\section{Introducción}

En el país, los sistemas constructivos rápidos y de bajo costo han tenido gran auge y con el crecimiento acelerado del sector, se ha dado un aumento en los productores y proveedores de estas modalidades de construcción. En cuanto a soluciones de vivienda, el principal desarrollo se dio en el método de prefabricado con baldosas y columnas de concreto, por lo que se hace una prioridad asegurar los debidos controles de calidad de los productos, de manera que se pueda asegurar un mínimo de resistencia y durabilidad de dichos elementos que procuren un mejor desempeño de las estructuras construidas con estos.

Hasta el momento en el país, no se ha desarrollado una normativa que regule la producción de este sistema de prefabricado, siendo este de vital importancia, ya que en marzo de 2010, el Instituto Nacional de Estadística y Censo, reportó que del total de construcciones de vivienda en el país, aproximadamente un $11 \%$ corresponde a este tipo de sistema. De este porcentaje, una parte importante se trata de bonos de la vivienda, ya que el sistema representa un ahorro aproximado para el cliente entre $15 \%$ y $20 \%$. En el campo de las construcciones destinadas a bien social, se destacan los datos del Banco Hipotecario de la Vivienda (BANVHI). Según los datos estadísticos presentados por esta institución [10], en el 2011 se extendieron 10461 bonos, de los cuales, 6786 fueron para construcción en lote propio y 1098 se trató de casos en los que se contempló el lote y la construcción de la estructura. Estos datos dan razón de la importancia de evitar que la calidad de este sistema constructivo no sea regulada de manera nacional.

Esta investigación trata de comparar dos métodos de ensayo para el control de la calidad para el sector industrial dedicado a esta actividad. Mediante una correlación estadísticamente válida, se puede obtener la validación del método de medición de la capacidad en flexión mediante carga en los tercios, comparado con el método de flexión en voladizo. Este último método ha sido el que tradicionalmente se ha utilizado para evaluar el desempeño de las columnas, ya que en cierta manera aproxima las condiciones de carga y de frontera del modelo analítico comúnmente utilizado para determinar el estado de fuerzas internas de la columna. Sin embargo, este método presenta una desventaja ya que requiere de equipo y de habilidad técnica mucho mayor que el de capacidad en flexión mediante carga aplicada en los tercios.

Para la investigación se evaluaron los productos de 5 fábricas productoras de columnas. Para cada fábrica, se fallaron 7 columnas con cada uno de los métodos. La correlación entre los resultados obtenidos permite determinar si existe o no diferencia significativa en los resultados de fuerzas internas en la columna (momentos de agrietamiento, momentos últimos) obtenidos con un método o con el otro.

\section{Sistema constructivo mediante columnas y baldosas prefabricadas de concreto}

Este sistema se basa en construcciones livianas que se utilizan en Europa, que fueron traídas como una opción más atractiva, por sus características de rapidez constructiva, modulación (evita el desperdicio) y facilidad de transporte. Se trata de un conjunto de secciones hechas en condiciones de planta de manufactura, compuestas por concreto prefabricado reforzado que se llevan de la planta al sitio de construcción y posteriormente son ensambladas mediante la modulación que disponga el fabricante. Cabe destacar que los procedimientos de cimentación y todo lo referente a configuración recomendada que requiere este tipo de secciones se especifica en el Código Sísmico de Costa Rica, CSCR [11].

\subsection{Tipos de columnas prefabricadas}

\subsubsection{Columnas prefabricadas de concreto, con refuerzo simple de acero.}

Estas se construyen mediante una armadura en acero estructural, que cumpla con las especificaciones ASTM A615 [4], ASTM A705 [5] ó ASTM A82 [6] según sea el caso de refuerzo sencillo o con capacidad de ser soldado y concreto que según las premisas de diseño cumpla con el momento requerido (ambos componentes diseñados según lo requiere el $\mathrm{ACl} 318$ [1]). Es importante recalcar que en algunos casos las columnas con refuerzo simple de acero armado, se conforman mediante uniones soldadas entre las barras internas, pues como se verá más adelante, esto puede repercutir en los resultados obtenidos con el método de ensayo con carga en los tercios. 
2.2.2 Columnas prefabricadas de concreto, con refuerzo pretensado de acero.

Estas se construyen mediante un alambre estructural conformado por torones de acero que presentan una configuración helicoidal alrededor del eje y que son tensados mediante pistones hidráulicos previo a la colocación y fragua del concreto, este alambre debe cumplir con la normas ASTM A416 [7], ASTM A421 [8] y ASTM A910 [9], y concreto que según las premisas de diseño cumpla con el momento requerido (ambos componentes diseñados según lo requiere el $\mathrm{ACl} 318$ [1]).

\subsection{Usos del sistema de construcción de prefabricado con baldosas y columnas}

Este sistema constructivo debido a su bajo costo, se utiliza principalmente para la construcción de viviendas de interés social. Además se utiliza para construcción de otras estructuras que presenten capacidad de modulación y en sistemas de división de propiedades, como tapias.

\subsection{Dimensiones comunes de las columnas prefabricadas de concreto}

Las dimensiones comunes para estas columnas se muestran en la Figura 1, junto con los valores numéricos en la Tabla 1. Estos valores provienen de estadísticas que combinan los datos recolectados de una amplia variedad de fabricantes de elementos de concreto prefabricado.
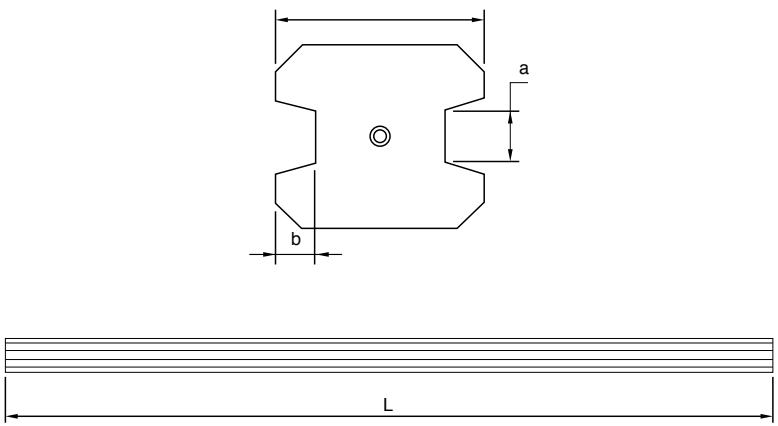

Figura 1. Esquema de las dimensiones de las columnas

\section{Metodología de análisis}

El sistema de la columna de concreto prefabricado, empotrada en su base, se modela basándose en las indicaciones del CSCR. En este, se especifica que la separación máxima entre columnas es de 1,5 $\mathrm{m}$ y entre las columnas se ubica una pared con $5 \mathrm{~cm}$ de espesor, con una
Tabla 1. Dimensiones comunes en las columnas prefabricadas de concreto reforzado

\begin{tabular}{|c|c|c|}
\hline Dimensión & Mínimo $(\mathrm{cm})$ & Máximo $(\mathrm{cm})$ \\
\hline Longitud de la columna (LA) & 315 & 330 \\
\hline Ancho de columna (A) & 12,0 & 14,0 \\
\hline Ancho de canal de la columna (a) & 3,5 & 5,0 \\
\hline Profundidad de canal de la columna (b) & 3,0 & 3,0 \\
\hline
\end{tabular}

altura libre de 2,75 m (H). Además, se asume que las paredes forman habitaciones de $6 \mathrm{~m}$ de ancho y tienen aleros de $1 \mathrm{~m}$ de ancho. A continuación se muestra el esquema de cuerpo libre en la columna cimentada con todas las cargas asociadas.

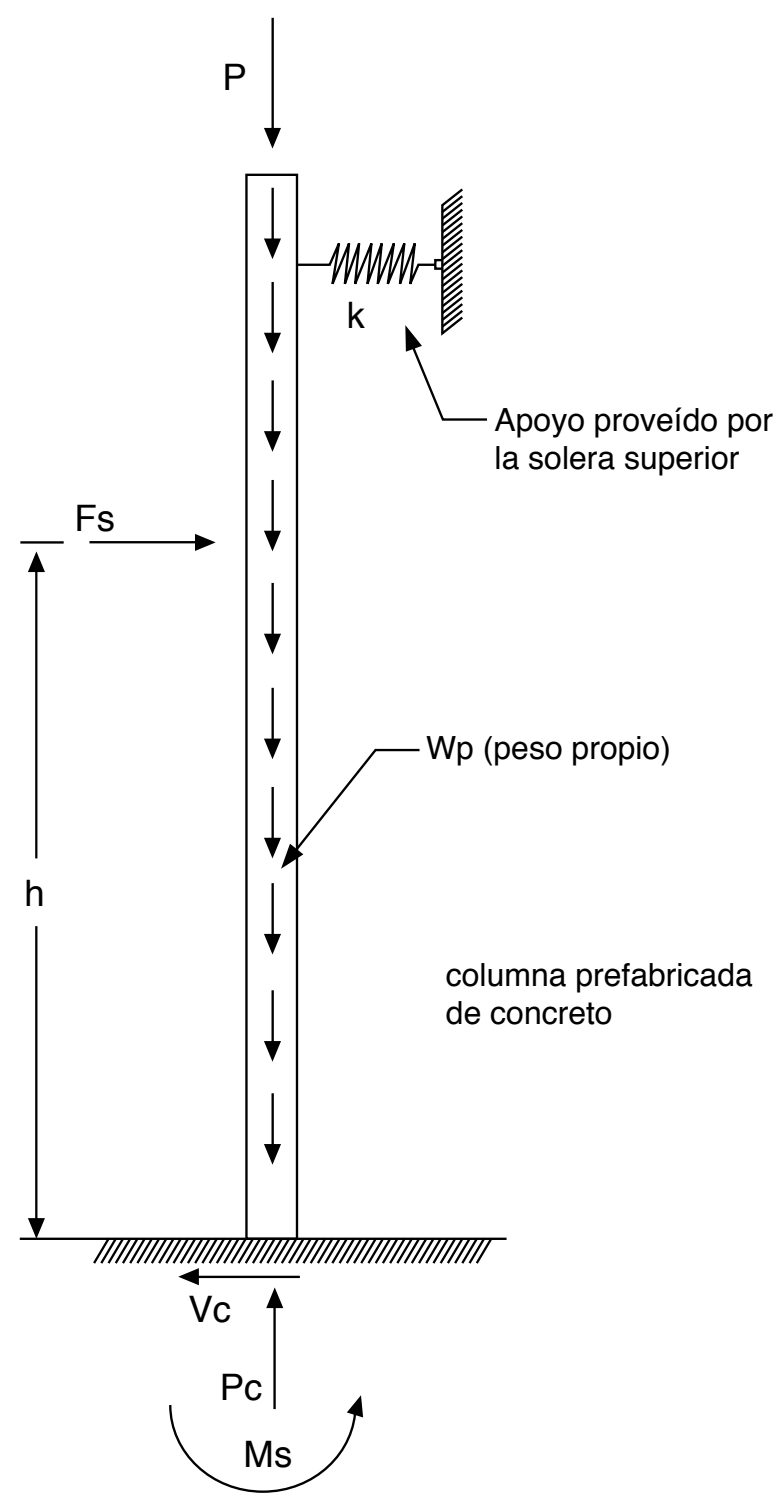

Figura 2. Diagrama de cargas asociadas a las columnas prefabricadas de concreto reforzado 
Donde:

Fs: fuerza sísmica.

P: carga vertical generada por el peso del techo.

wp: peso propio de la pared.

Ms: momento sísmico de vuelco.

Vc: fuerza cortante en la base de la columna.

Pc: carga axial en la base de la columna.

A continuación se muestra la estructuración que exige el CSCR con respecto al sistema constructivo:

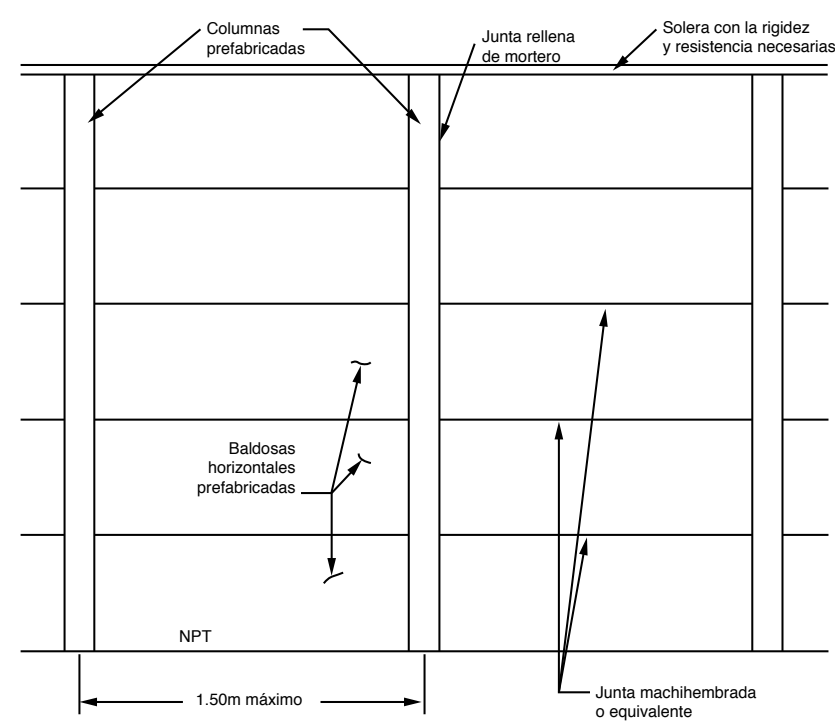

Figura 3. Requerimientos del Código Sísmico de Costa Rica sobre la estructuración del sistema constructivo

Fuente: Colegio Federado de Ingenieros y Arquitectos (2010). Código Sísmico de Costa Rica CSCR-2010 (17/16). Editoral Tecnológica de Costa Rica [11].

\section{Métodos de ensayo para determinar la capacidad en flexión de las columnas de concreto prefabricado}

Para determinar la capacidad en flexión de una sección el ensayo más común y conveniente por la facilidad de montaje y aplicación de la carga, es el ensayo de flexión con cargas aplicadas en los tercios. Con este tipo de ensayo es posible evaluar la sección central del espécimen sometido a momento puro. Sin embargo, dentro del marco de la mecánica de los materiales, no solo es importante investigar el comportamiento de la sección ante la carga aplicada, sino que también es de suma importancia asemejar las condiciones de servicio del elemento que se está investigando.

Para los dos métodos mostrados en esta investigación, la velocidad de carga utilizada fue la misma. En ambos casos, la columna fue cargada a una razón constante de $16 \mathrm{~N} / \mathrm{s}$, este valor se desarrolló a partir del análisis de la condición de carga usada tradicionalmente, donde se aplicaba manualmente una carga incremental, mediante una bomba hidráulica conectada al gato hidráulico. El valor de velocidad utilizado, corresponde al promedio de las pendientes de las rectas de mejor ajuste de gráficos experimentales similares al mostrado en la figura 4.

Para ambos métodos, se deben realizar mediciones de desplazamiento. Para el método de voladizo se realiza en intervalos $200 \mathrm{~N}$ hasta alcanzar los $2200 \mathrm{~N}$. Como se discutirá más adelante es posible demostrar que la carga que produce, en el ensayo de carga en los tercios, un momento de igual magnitud al generado en el ensayo en voladizo, es aproximadamente 3 veces mayor para las condiciones de esta investigación. Por esta razón los intervalos de toma de carga para el ensayo de carga en los tercios corresponden $250 \mathrm{~N}$ hasta alcanzar $6000 \mathrm{~N}$.

En el momento que se alcanza el valor de carga máxima hasta el cual se deben tomar mediciones de desplazamiento según el tipo ensayo, se debe retirar el deformímetro y continuar la carga hasta registrar el máximo. Durante el proceso de carga, se debe observar la sección de la columna que tiene el momento flector máximo (en voladizo, la sección de empotramiento y en los tercios, la sección central de la columna) para determinar el momento en el que se inicia el agrietamiento de la sección. La carga de grieta se define como aquella en la que se produce una grieta en la zona de momento máximo, con una abertura mayor de $0.3 \mathrm{~mm}$. Los intervalos de toma de datos, el criterio para la definición de carga de grieta, la velocidad y la carga máxima en la cual se detiene la toma de datos de desplazamiento, corresponden a parámetros utilizados tradicionalmente en el ensayo de estos especímenes mediante el método de voladizo y de la experiencia se ha demostrado que logran una buena descripción del comportamiento de la columna.

Cuando no se tiene un dispositivo con algún tipo de servocontrol que produzca aumentos continuos de carga, se puede hacer cada incremento en un tiempo entre 5 y 7 segundos, con pausas de entre 5 y 7 segundos para la toma de datos. Este fenómeno de intervalos de carga se expresa gráficamente en la Figura 4 . La línea recta que se muestra en la figura 4 , corresponde a una carga aplicada de manera continua con una velocidad constante $16 \mathrm{~N} / \mathrm{s}$, que a su vez corresponde a la línea de mejor ajuste para los intervalos descritos anteriormente. La toma de datos de desplazamiento finaliza cuando se alcanza la carga máxima definida para cada método o cuando los incrementos de carga son insignificantes. El ensayo se debe continuar 


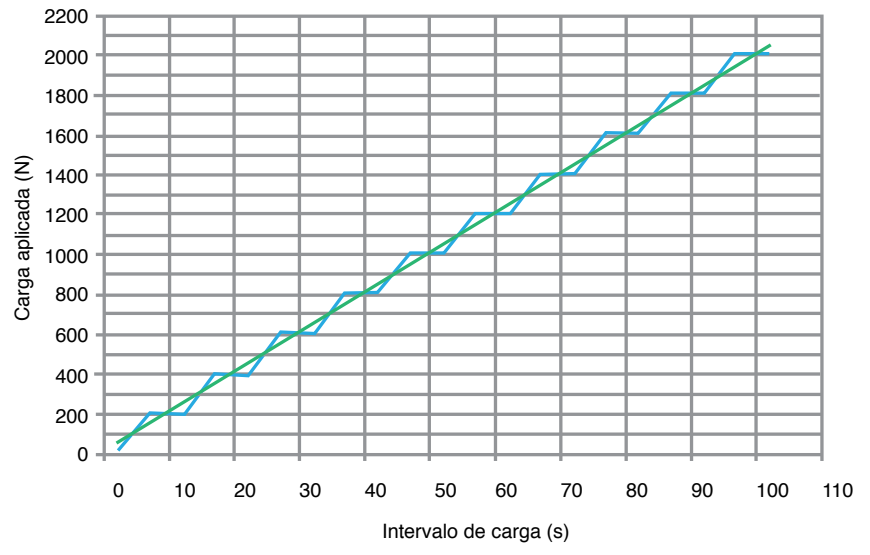

Figura 4. Emulación de la carga constante mediante incrementos de $200 \mathrm{~N}$

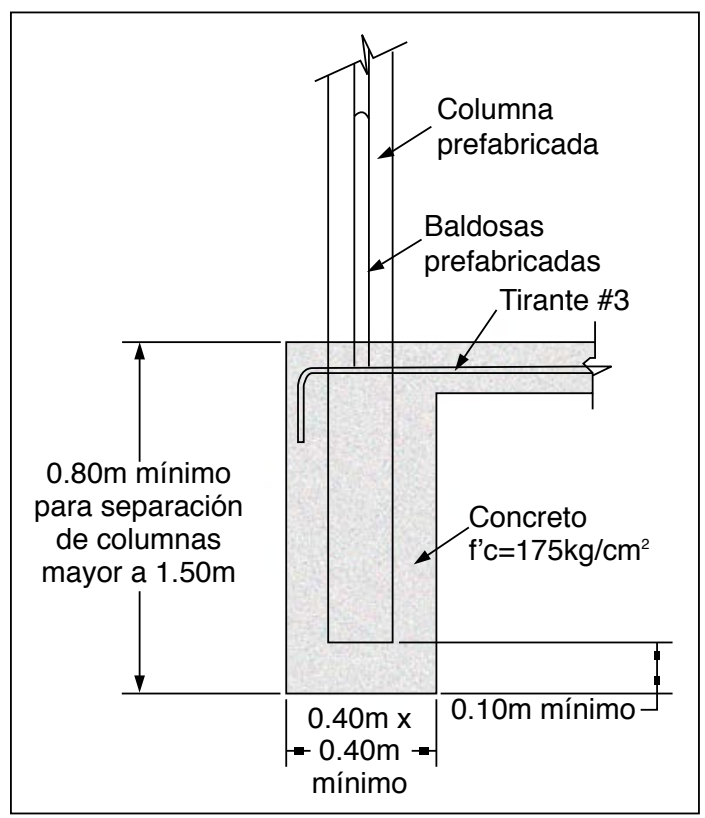

Figura 5. Configuración de la cimentación según el Código Sísmico de Costa Rica [11]

Fuente: Colegio Federado de Ingenieros y Arquitectos (2010). Código Sísmico de Costa Rica CSCR-2010 (17/7). Editoral Tecnológica de Costa Rica

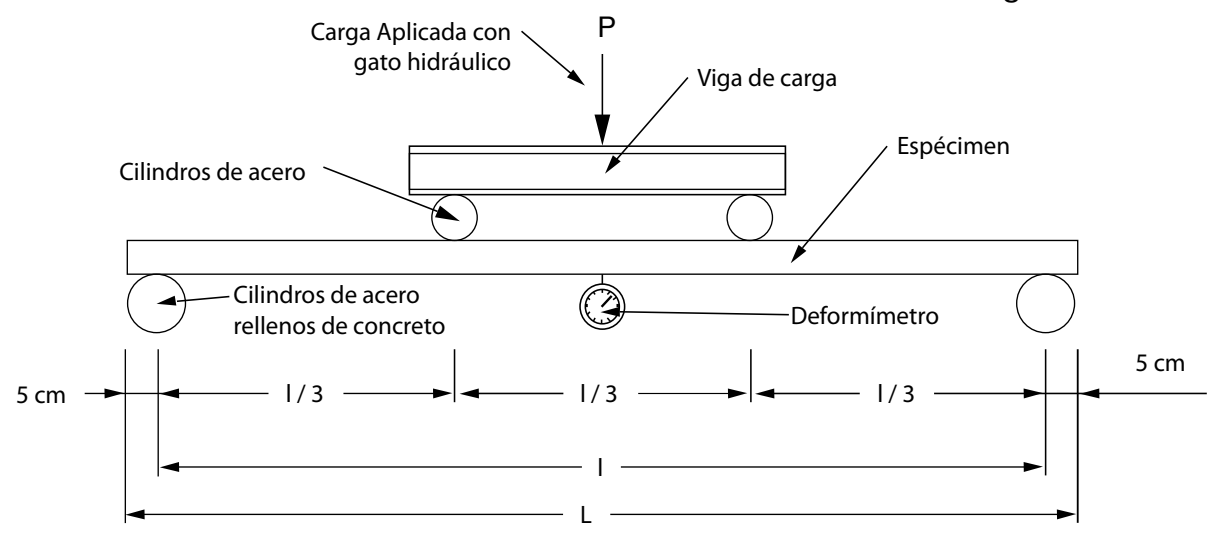

aplicando una carga de manera continua, procurando alcanzar la carga máxima en un tiempo de 1 a 3 minutos, esta duración, corresponde a un intervalo promedio en el que tradicionalmente columnas de concreto ensayadas por el método de voladizo, ha demorado en alcanzar la falla cuando el operador realiza una aplicación de carga continua.

Como se muestra en la Figura 5, la forma constructiva según el CSCR, especifica que las columnas se encuentran empotradas en la base, donde la distancia de empotramiento es equivalente a la distancia de cimentación, por lo que una prueba semejando esta condición se hace muy atractiva para determinar la capacidad real de la sección crítica en estudio (base del empotramiento en la columna). Sin embargo, si el diseño de estos elementos es regido por su resistencia a la flexión, una prueba que evalúe la sección sometida a momento puro es más representativa. A continuación se explica en detalle la metodología de falla de cada uno de los métodos y las diferencias más relevantes que presentan uno sobre el otro.

\subsection{Ensayo de capacidad en flexión mediante cargas aplicadas en los tercios.}

Este ensayo se inicia determinando las dimensiones del espécimen de ensayo. Luego, la columna se pesa. A continuación se coloca sobre dos apoyos cilíndricos, tal y como se muestra en la Figura 6 y se procede a colocar los cilindros y la viga de acero que transmiten la carga proveniente del gato hidráulico en los tercios del espécimen de ensayo.

Sobre la columna se instala un deformímetro mediante el cual se determina el desplazamiento vertical que sufre el espécimen en los incrementos de carga descritos en la sección 4.

Una vez que el deformímetro es instalado correctamente en el centro del claro, se inicia la aplicación continua de carga. Las lecturas de desplazamiento se registran en los

Figura 6. Configuración para el ensayo de capacidad en flexión mediante cargas aplicadas en los tercios 


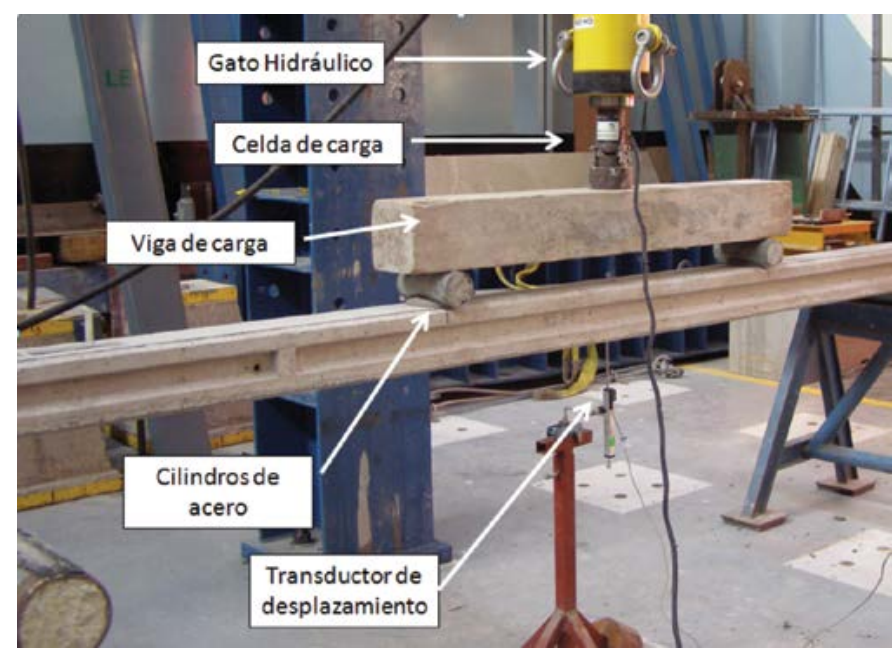

Figura 7. Fotografía del montaje utilizado en el ensayo de capacidad en flexión mediante cargas aplicadas en los tercios

incrementos de fuerza determinados en la sección 4 , se registra el valor de carga que produce el agrietamiento de la sección determinado según se describe en la sección 4 y se continua con la aplicación de carga hasta que se registre un máximo de carga.

\subsubsection{Análisis de la capacidad mediante cargas aplicadas en} los tercios

El momento de agrietamiento y el momento máximo se deben calcular utilizando la ecuación 1 :

$$
M=\left(P+P_{\text {apar }}\right) * \frac{(I)}{6}+M_{p p}
$$

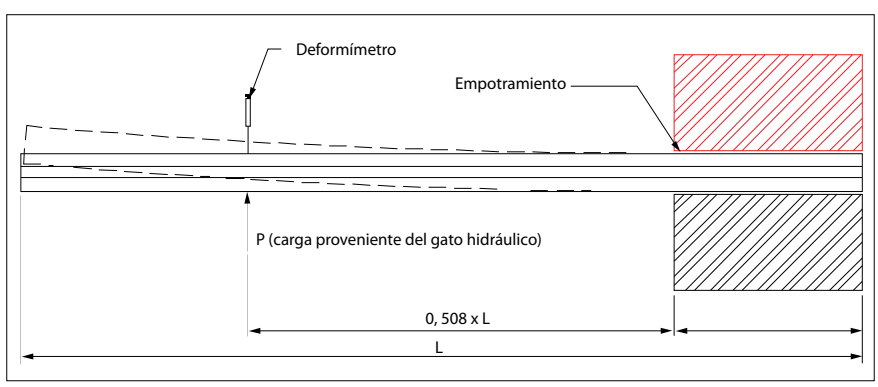

Figura 8. Configuración para el ensayo de capacidad en flexión mediante carga aplicada en voladizo
Donde:

$\mathrm{M}$ es el momento en el tercio medio de la columna $[\mathrm{N} \cdot \mathrm{m}]$.

I es la distancia entre los apoyos para el espécimen [m]

L es la longitud total de la columna [m]

$\mathrm{P}$ es la carga que se registra en los instrumentos de medición del ensayo $[\mathrm{N}]$.

Papar es el peso que aportan los aparejos utilizados sobre el espécimen para una adecuada aplicación de la carga en el ensayo [N].

MPP es el momento que se genera por el peso propio de la columna sobre el centro de la misma, se debe calcular mediante la ecuación [N·m]:

$$
\mathrm{M}_{\mathrm{pp}}=\frac{(\mathrm{I})^{2}}{8} * \frac{\mathrm{W}_{\mathrm{c}}}{\mathrm{L}}
$$

Donde:

Wc corresponde al peso propio de la columna [N].

\subsection{Ensayo de capacidad en flexión en voladizo}

Se determinan las dimensiones y peso del espécimen como en el caso anterior. Posteriormente, se mide y se marca la distancia de empotramiento que corresponde a $0,22 \mathrm{~L}$ y el punto de aplicación de la carga que corresponde a 0,508 L, ver (figura 8). La muestra se coloca sobre la máquina de ensayo encargada de aplicar la carga de empotramiento. Esta máquina ejerce una presión constante, tradicionalmente en la ejecución de este ensayo se ha utilizado un valor de carga de 28,5 $\pm 1,5 \mathrm{kN}$ sobre la longitud de empotramiento. Finalmente se procede a colocar el gato hidráulico y la celda de carga en el punto previamente determinado.

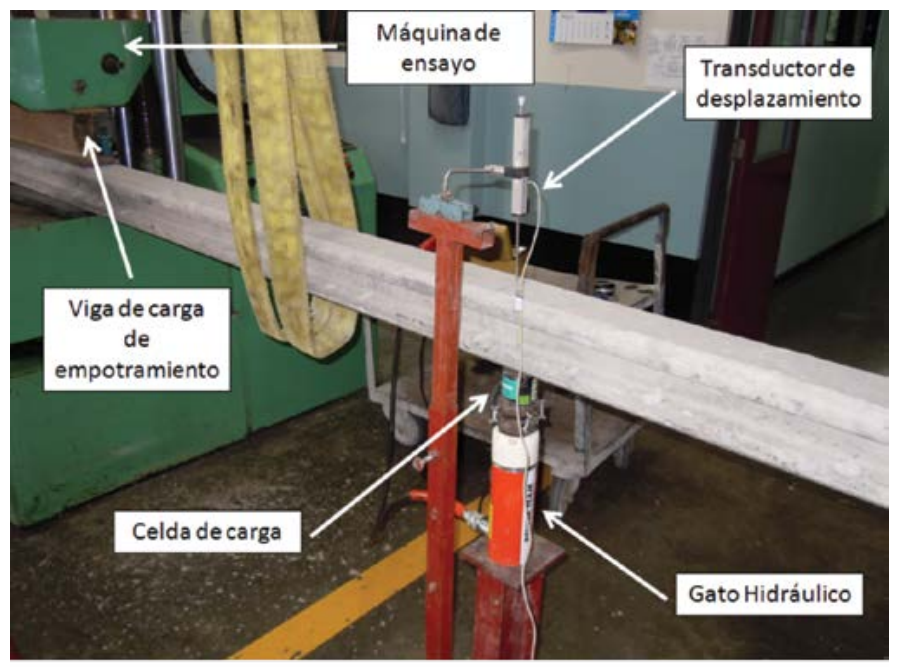

Figura 9. Fotografía del montaje utilizado en el ensayo de capacidad en flexión mediante carga aplicada en voladizo 
Se instala un deformímetro sobre la columna y alineado con el gato hidráulico, que determina el desplazamiento vertical que sufre el espécimen para cada incremento de carga descrito en la sección 4.

Una vez que el deformímetro es instalado correctamente, se inicia la aplicación continua de carga. Las lecturas de desplazamiento se registran en los incrementos de fuerza determinados en la sección 4 , se registra el valor de carga que produce el agrietamiento de la sección determinado según se describe en la sección 4 y se continúa con la aplicación de carga hasta que se registre un máximo de carga.

\subsubsection{Análisis de la capacidad en flexión en voladizo}

El momento de agrietamiento y el momento máximo se deben calcular utilizando la siguiente ecuación:

$$
\mathrm{M}=\mathrm{P}_{\text {eq }} \bullet 0.508 \bullet \mathrm{L}
$$

Donde:

$\mathrm{M}$ es el momento en la base de la columna [N.m].

Peq es una carga equivalente para un sistema en voladizo en donde se corrige el efecto del peso propio de la columna. Esta se debe calcular utilizando la siguiente ecuación [N]:

$$
\text { Peq }=P-0,03 \bullet W_{c}
$$

Donde:

$P$ es la carga que se registra en los instrumentos de medición del ensayo $[\mathrm{N}]$.

Wc corresponde al peso propio de la columna $[\mathrm{N}]$.

El efecto que tiene el concepto de carga equivalente sobre el procedimiento de ensayo, es una deformación inicial del espécimen que se mantiene en un rango elástico de deformaciones, sin embargo, el efecto de esa deformación inicial se compensa mediante un análisis de compatibilidad de las deformaciones, en el que se establece que a la carga registrada por el equipo se le debe restar una fracción del peso propio para eliminar el efecto de la carga inicial.

Tabla 2. Análisis de varianza de las muestras, según el método de falla y la fase

\begin{tabular}{|c|c|c|c|c|c|c|c|c|}
\hline Ensayo & \multicolumn{4}{|c|}{ Voladizo } & \multicolumn{4}{|c|}{ Tercios Medios } \\
\hline Fase & \multicolumn{2}{|c|}{ Agrietamiento } & \multicolumn{2}{|c|}{ Falla } & \multicolumn{2}{|c|}{ Agrietamiento } & \multicolumn{2}{|c|}{ Falla } \\
\hline \multirow{2}{*}{ Estadístico } & $F_{\text {Levene }}$ & $F_{c r(0,05,4,48)}$ & $F_{\text {Levene }}$ & $\mathrm{F}_{\mathrm{cr}(0,0,5,4,27)}$ & $F_{\text {Levene }}$ & $F_{c r(0,05,4,30)}$ & $\mathrm{F}_{\text {Levene }}$ & $\mathrm{F}_{\mathrm{cr}(0,0,95,4,30)}$ \\
\hline & 0,523 & 2,928 & 1,003 & 2,728 & 0,227 & 2,690 & 0,342 & 2,690 \\
\hline$S_{A}{ }^{2}=S_{B}{ }^{2}=S_{C}{ }^{2}=S_{D}{ }^{2}=S_{E}^{2}$ & \multicolumn{2}{|c|}{ Se cumple } & \multicolumn{2}{|r|}{ mple } & \multicolumn{2}{|c|}{ Se cumple } & \multicolumn{2}{|c|}{ Se cumple } \\
\hline
\end{tabular}
de ensayo

\section{Programa experimental}

\subsection{Muestreo de especímenes}

Los especímenes fueron muestreados directamente de la planta de producción. Las visitas se realizaron en fechas programadas en las que la planta se encontraba en producción, para ejecutar el muestreo sobre todo el lote manufacturado.

Para obtener una alta confiabilidad de los resultados, se procedió a muestrear 14 columnas por fabricante $(7$ por método de falla), ya que experimentalmente para una confianza del $95 \%$, la incertidumbre (específicamente la $\mathrm{t}$ de student asociada), tiene variaciones consideradas insignificantes en la práctica después de la sétima observación si se supone una distribución normal de los resultados.

\subsection{Metodología de investigación}

Se dividieron las 14 columnas en dos grupos de siete de manera aleatoria. Cada uno de los grupos fue ensayado con uno de los dos métodos. De esta manera, mediante cálculo estructural, se obtuvo la capacidad máxima en flexión de los elementos y se determinó la diferencia de valores que arrojan los resultados entre las dos metodologías de falla. Luego se compararon los resultados para encontrar una correlación entre los métdos.

Los resultados de este estudio fueron tratados mediante los procedimientos que se describen en la norma ASTM E178 [3], sobre el tratamiento de observaciones atípicas.

Se aplicaron según las recomendaciones de la norma para los comportamientos de valores extremos, para una sola observación y para varias. El método más utilizado fue el análisis de Tietjen - Moore, que permite el análisis de más de dos valores atípicos según el tamaño de la muestra que se está trabajando.

\subsection{Resultados experimentales}

Dado que cada uno de los fabricantes tiene sus técnicas de manufactura, es importante al momento de generar resultados inclusivos para la generalidad de los fabricantes de estos elementos, tomar en cuenta estas diferencias mediante el análisis de varianza. El mismo se realizó mediante la metodología de Levene, los resultados estadísticos se muestran en la Tabla 2. 
Tabla 3. Resumen de resultados del momento para los ensayos de capacidad en flexión mediante la aplicación de carga en voladizo y en los tercios; para el momento de agrietamiento y el máximo momento de la falla

\begin{tabular}{|c|c|c|c|c|c|}
\hline Fabricante & $\begin{array}{l}\text { Momento en método del } \\
\text { voladizo }\left(\mathrm{N}^{*} \mathrm{~m}\right)\end{array}$ & $\begin{array}{l}\text { Momento en método } \\
\text { demedios tercios }\left(\mathrm{N}^{\star} \mathrm{m}\right)\end{array}$ & $\begin{array}{l}\text { Diferencia entre métodos } \\
\qquad\left(\mathrm{N}^{*} \mathrm{~m}\right)\end{array}$ & Diferencia entre métodos (\%) & Tipo de refuerzo \\
\hline \multirow{8}{*}{ A } & M agrietamiento & M agrietamiento & $\Delta \mathrm{M}$ agrietamiento & $\Delta \mathrm{M}$ agrietamiento & \multirow{8}{*}{ Refuerzo sencillo } \\
\hline & 2693 & 3188 & \multirow{3}{*}{$\begin{array}{r}496 \\
-\quad-4\end{array}$} & \multirow{3}{*}{${ }^{-} 18,41 \%$} & \\
\hline & $s$ agrietamiento & $\mathrm{s}$ agrietamiento & & & \\
\hline & 149 & 249 & & & \\
\hline & $M$ falla & $M$ falla & $\Delta \mathrm{M}$ falla & $\Delta \mathrm{M}$ falla & \\
\hline & 3796 & 3826 & \multirow{3}{*}{$\begin{array}{l}30 \\
-\end{array}$} & \multirow{3}{*}{ \% } & \\
\hline & s falla & s falla & & & \\
\hline & 471 & 149 & & & \\
\hline \multirow{8}{*}{ B } & M agrietamiento & M agrietamiento & $\Delta \mathrm{M}$ agrietamiento & $\Delta \mathrm{M}$ agrietamiento & \multirow{8}{*}{ Refuerzo pretensado } \\
\hline & 2877 & 2543 & \multirow{3}{*}{${ }^{-} \quad 334$} & \multirow{3}{*}{$-\quad \%$} & \\
\hline & $\mathrm{s}$ agrietamiento & $\mathrm{s}$ agrietamiento & & & \\
\hline & 240 & 200 & & & \\
\hline & $M$ falla & $M$ falla & $\Delta \mathrm{M}$ falla & $\Delta \mathrm{M}$ falla & \\
\hline & 4775 & 5250 & \multirow{3}{*}{$\begin{array}{l}475 \\
-\end{array}$} & \multirow{3}{*}{$\begin{array}{c}\% \\
-\end{array}$} & \\
\hline & s falla & s falla & & & \\
\hline & $\overline{1032}$ & 192 & & & \\
\hline \multirow{8}{*}{ c } & M agrietamiento & M agrietamiento & $\Delta \mathrm{M}$ agrietamiento & $\Delta \mathrm{M}$ agrietamiento & \multirow{8}{*}{$\begin{array}{l}\text { Refuerzo sencillo, } \\
\text { susceptible a fallapor } \\
\text { fragilidad en la unión soldada }\end{array}$} \\
\hline & 4053 & 2178 & \multirow{3}{*}{$\begin{array}{r}1876 \\
-\quad-1\end{array}$} & \multirow{3}{*}{$\begin{array}{r}\% \\
-\quad \%\end{array}$} & \\
\hline & $\mathrm{s}$ agrietamiento & $\mathrm{s}$ agrietamiento & & & \\
\hline & 69 & 390 & & & \\
\hline & $M$ falla & $M$ falla & $\Delta \mathrm{M}$ falla & $\Delta \mathrm{M}$ falla & \\
\hline & 4911 & 2572 & \multirow{3}{*}{$\begin{array}{l}2339 \\
-\end{array}$} & \multirow{3}{*}{$\begin{array}{c}\% \\
-\end{array}$} & \\
\hline & s falla & s falla & & & \\
\hline & 329 & 425 & & & \\
\hline \multirow{8}{*}{ D } & M agrietamiento & M agrietamiento & $\Delta \mathrm{M}$ agrietamiento & $\Delta \mathrm{M}$ agrietamiento & \multirow{8}{*}{$\begin{array}{l}\text { Refuerzo sencillo, } \\
\text { susceptible a fallapor } \\
\text { fragilidad en la unión soldada }\end{array}$} \\
\hline & 1530 & 1265 & \multirow{3}{*}{$-\quad 265$} & \multirow{3}{*}{$\%$} & \\
\hline & $\mathrm{s}$ agrietamiento & $\mathrm{s}$ agrietamiento & & & \\
\hline & 182 & 180 & & & \\
\hline & $M$ falla & $M$ falla & $\Delta \mathrm{M}$ falla & $\Delta \mathrm{M}$ falla & \\
\hline & 3161 & 2491 & \multirow{3}{*}{$\begin{array}{l}669 \\
-\end{array}$} & \multirow{3}{*}{$\begin{array}{c}- \\
\quad \% \\
-\end{array}$} & \\
\hline & s falla & s falla & & & \\
\hline & 542 & 228 & & & \\
\hline \multirow{8}{*}{$\mathrm{E}$} & M agrietamiento & M agrietamiento & $\Delta \mathrm{M}$ agrietamiento & $\Delta \mathrm{M}$ agrietamiento & \multirow{8}{*}{ Refuerzo pretensado } \\
\hline & 2501 & 2760 & - & & \\
\hline & $\mathrm{s}$ agrietamiento & $\mathrm{s}$ agrietamiento & 259 & $\%$ & \\
\hline & 76 & 413 & & & \\
\hline & M falla & $M$ falla & $\Delta \mathrm{M}$ falla & $\Delta \mathrm{M}$ falla & \\
\hline & 4802 & 5027 & & - & \\
\hline & s falla & s falla & 225 & $\%$ & \\
\hline & 111 & 605 & & & \\
\hline
\end{tabular}

La prueba o metodología de Levene corresponde a un parámetro estadístico que mide la diferencia entre las varianzas y la probabilidad de haberla obtenido al azar bajo el supuesto de que las varianzas poblacionales de los grupos sean iguales.
En la Tabla 3, se muestra el resumen de resultados de las pruebas practicadas sobre los especímenes. Se presentan los parámetros más importantes de diseño como lo son el momento de agrietamiento y el momento de falla. La primera columna muestra el fabricante, que se expresa 
Tabla 4. Relaciones de momento entre las metodologías de falla (desarrollo completo con análisis de datos atípicos sobre las observaciones, pero no entre fabricantes)

\begin{tabular}{|c|c|c|}
\hline Fabricante & Relación $\mathbf{M}_{\mathrm{a}, \mathrm{v}} / \mathbf{M}_{\mathrm{a}, \mathrm{t}}$ & Relación $\mathbf{M}_{\mathrm{f}, \mathrm{v}} / \mathbf{M}_{\mathrm{f}, \mathrm{t}}$ \\
\hline $\mathrm{A}$ & 0,84 & 0,99 \\
\hline $\mathrm{B}$ & 1,13 & 0,91 \\
\hline $\mathrm{C}$ & 1,94 & 1,91 \\
\hline $\mathrm{D}$ & 1,21 & 1,27 \\
\hline $\mathrm{E}$ & 0,91 & 0,96 \\
\hline Promedio & 1,21 & 1,21 \\
\hline Desviación estándar & 0,39 & 0,37 \\
\hline
\end{tabular}

como una letra, la segunda y tercera muestran la capacidad de la sección de columna cuando se somete al ensayo de carga en voladizo y cargas en los tercios, respectivamente, con su respectivo momento de agrietamiento, momento de falla y las desviaciones estándar asociadas. En la cuarta y quinta columna se muestra la diferencia que existe entre los métodos tanto en $\mathrm{N} \cdot \mathrm{m}$, como en porcentaje (proporción al momento mediante el ensayo de carga en el voladizo, ya que este es el que se ha utilizado tradicionalmente). En la sexta columna se muestra el tipo de refuerzo con el que contaba el elemento, el cual fue caracterizado después de la falla, según lo que se observó en la metodología constructiva del espécimen y el mecanismo de falla de la sección.

$M=$ momento promedio.

$\mathrm{s}=$ desviación estándar.

En la tabla 3, se muestran los parámetros de capacidad en flexión de cada uno de los fabricantes. Cabe destacar que los datos presentados corresponden a los datos ajustados según la norma ASTM E178, donde según la tabla 2, se puede observar que las varianzas entre las muestras no presentan diferencias significativas. En la tabla 4, se muestra el resumen del cálculo de relaciones entre el método de capacidad en flexión mediante carga aplicada en voladizo y el método de capacidad en flexión mediante carga aplicada en los tercios, para cada una de las fases de la prueba.

* Ma,v se refiere al momento de agrietamiento en la prueba con carga aplicada al voladizo, Ma,t se refiere al momento de agrietamiento en la prueba con carga aplicada en los tercios.

${ }^{*} \mathrm{Mf}, \mathrm{v}$ se refiere al momento de falla en la prueba con carga aplicada al voladizo, Mf,t se refiere al momento de falla en la prueba con carga aplicada en los tercios.

En los datos obtenidos en la Tabla 4, se observa diferencias respecto a la media grupal para el fabricante $\mathrm{C}$, de 0,73.
Tabla 5. Relaciones de momento entre las metodologías de falla (con análisis de datos atípicos entre fabricantes)

\begin{tabular}{|c|c|c|}
\hline Fabricante & Relación $\mathbf{M}_{\mathrm{a}, \mathrm{v}} / \mathbf{M}_{\mathrm{a}, \mathrm{t}}$ & Relación $\mathbf{M}_{\mathrm{t}, \mathrm{v}} / \mathbf{M}_{\mathrm{t}, \mathrm{t}}$ \\
\hline $\mathrm{A}$ & 0,84 & 0,99 \\
\hline $\mathrm{B}$ & 1,13 & 0,91 \\
\hline $\mathrm{D}$ & 1,21 & 1,27 \\
\hline $\mathrm{E}$ & 0,91 & 0,96 \\
\hline Promedio & 1,02 & 1,03 \\
\hline Desviación estándar & 0,15 & 0,14 \\
\hline
\end{tabular}

Este resultado indica que existe un $73 \%$ de diferencia entre los métodos de voladizo y de carga en los tercios en los resultados obtenidos para el fabricante $\mathrm{C}$. Esta alza de la media, provoca que inclusive el fabricante $D$ se presente como un resultado disperso, por lo que para estudiar la presencia de algún fenómeno atípico, se analizan los datos mediante la metodología para observaciones atípicas de Grubbs que da negativa para que ambos valores sean observaciones atípicas. Se recurre a corroborar que el valor extremo del fabricante $C$, no sea una observación atípica, mediante la metodología de Dixon; en este caso la observación es descrita como atípica y se procede a generar los resultados sin la participación del fabricante $\mathrm{C}$, con lo que se genera la Tabla 5 .

De igual manera, se procedió a generar los resultados sobre los datos base, sin ningún tipo de tratamiento estadístico, ya que es importante destacar que los procedimientos estadísticos son una herramienta para generar información sin las problemáticas que traen los valores atípicos en un ensayo (en un $95 \%$ de los casos estos valores están ausentes en la prueba), sin embargo es importante mostrar la totalidad de los resultados donde se puede evidenciar el impacto que tiene un método constructivo particular en la distribución de resultados. En la Tabla 6, se muestra el resumen del cálculo con los datos base.

Tabla 6. Relaciones de momento entre las metodologías de falla (desarrollo completo sin análisis de valores atípicos sobre las observaciones o entre fabricantes)

\begin{tabular}{|c|c|c|}
\hline Fabricante & Relación $\mathbf{M}_{\mathrm{a}, \mathrm{v}} / \mathbf{M}_{\mathrm{a}, \mathrm{t}}$ & Relación $\mathbf{M}_{\mathrm{f}, \mathrm{v}} / \mathbf{M}_{\mathrm{t}, \mathrm{t}}$ \\
\hline $\mathrm{A}$ & 0,84 & 0,99 \\
\hline $\mathrm{B}$ & 0,99 & 0,91 \\
\hline $\mathrm{C}$ & 1,94 & 1,91 \\
\hline $\mathrm{D}$ & 1,32 & 1,27 \\
\hline $\mathrm{E}$ & 0,99 & 0,93 \\
\hline Promedio & 1,22 & 1,20 \\
\hline Desviación estándar & 0,39 & 0,38 \\
\hline
\end{tabular}


Es importante recalcar que los resultados de las Tabla 4, Tabla 5 y Tabla 6 , no se refieren a la capacidad de las secciones, un valor alto en la relación de sus momentos, quiere decir que resiste el momento aplicado en una proporción mayor en el ensayo de capacidad en flexión mediante el método de carga en voladizo, respecto a la carga en el tercio medio y viceversa. Para corroborar capacidades de las secciones, se debe acudir a la Tabla 2, donde se presenta el análisis estadístico desglosado con las respectivas diferencias, para todos los fabricantes, en los dos métodos de ensayo, según las fases de prueba.

Como se ha mencionado anteriormente, para los fabricantes C y D se presenta una relación atípica entre en los valores de momento de agrietamiento y de momento de falla que se aleja de los resultados para los demás fabricantes. A partir de los resultados que se muestran en tabla 3 , se

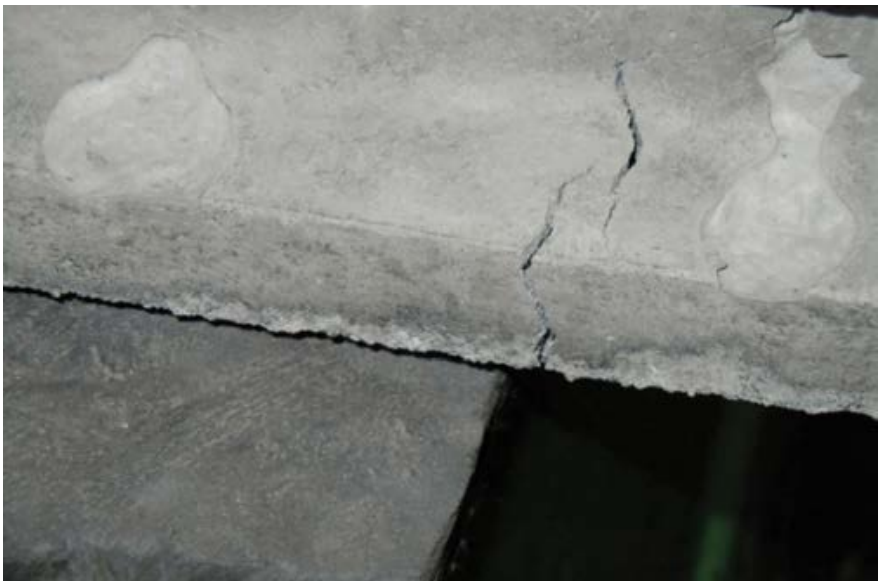

Figura 10. Falla de columna pre-esforzada en flexión mediante carga aplicada en voladizo

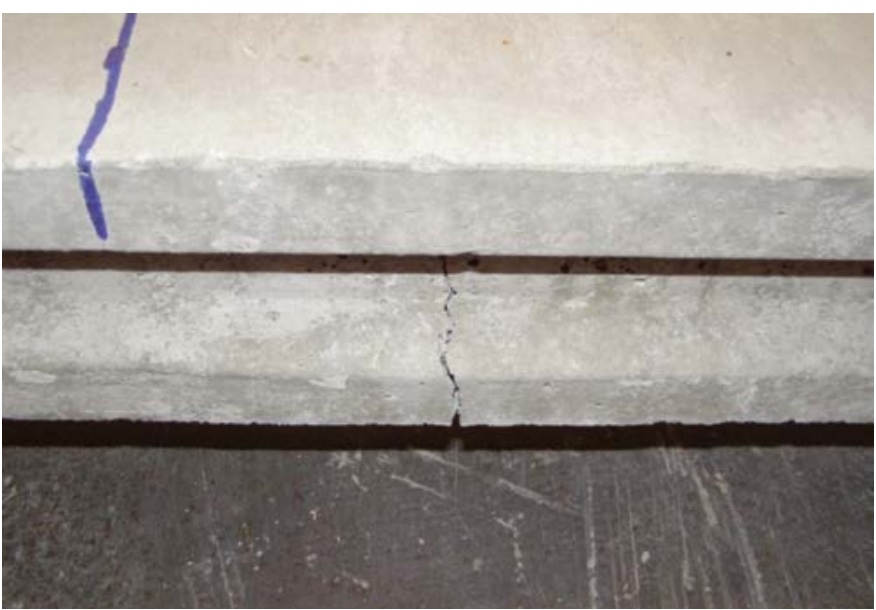

Figura 11. Falla de columna reforzada en flexión mediante carga aplicada en voladizo puede verificar que la relación entre los métodos para la fabricantes $C$ y $D$ toma un valor muy diferente al de los otros fabricantes dado que los momentos de agrietamiento y falla obtenidos con el método de carga en los tercios es significativamente menor al obtenido en la falla con el método de voladizo. Durante la ejecución de los ensayos se observó que las fallas de las columnas ensayas con el método de voladizo se presentaron en la sección cercana al empotramiento indiferentemente del fabricante, iniciando con una grieta en la cara inferior. En las figuras 10 y 11 se observa el agrietamiento típico que se presentó en los especímenes.

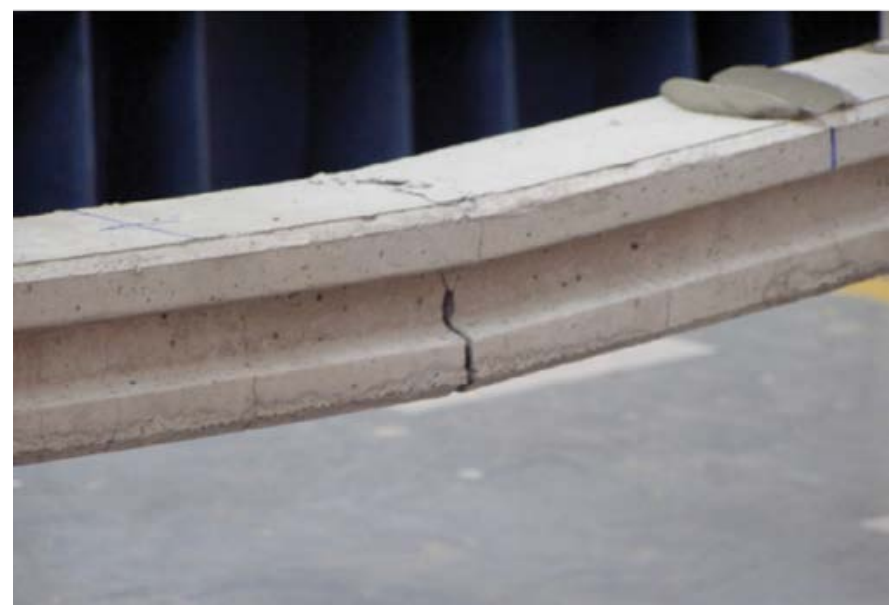

Figura 12. Agrietamiento en la sección central de la columna ensayada con el método de cargas en los tercios

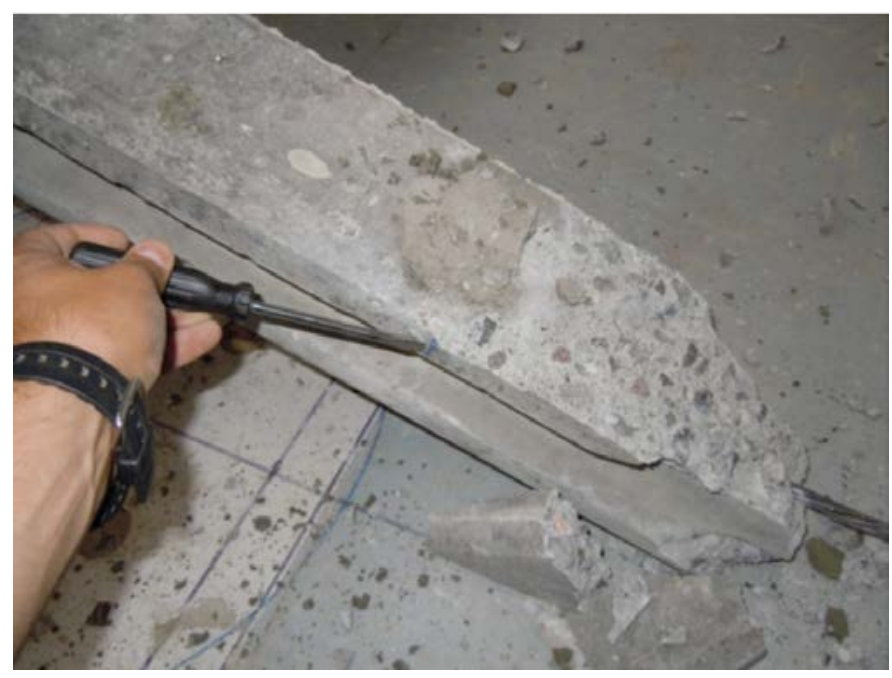

Figura 13. Falla de columna ensayada con el método de cargas en los tercios por qgrietamiento en el tramo central

Con respecto a las fallas obtenidas utilizando el método de carga en los tercios, en las figuras 12 y 13 se muestran las fallas típicas de las muestras en las cuales no se presentó 


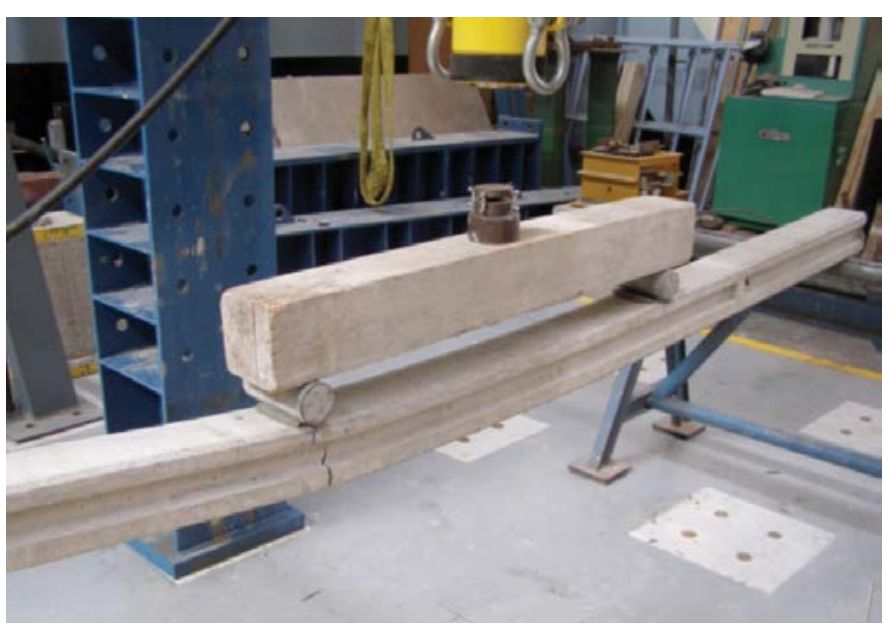

Figura 14. Agrietamiento en punto de aplicación de carga en columna ensayada con el método de cargas en los tercios

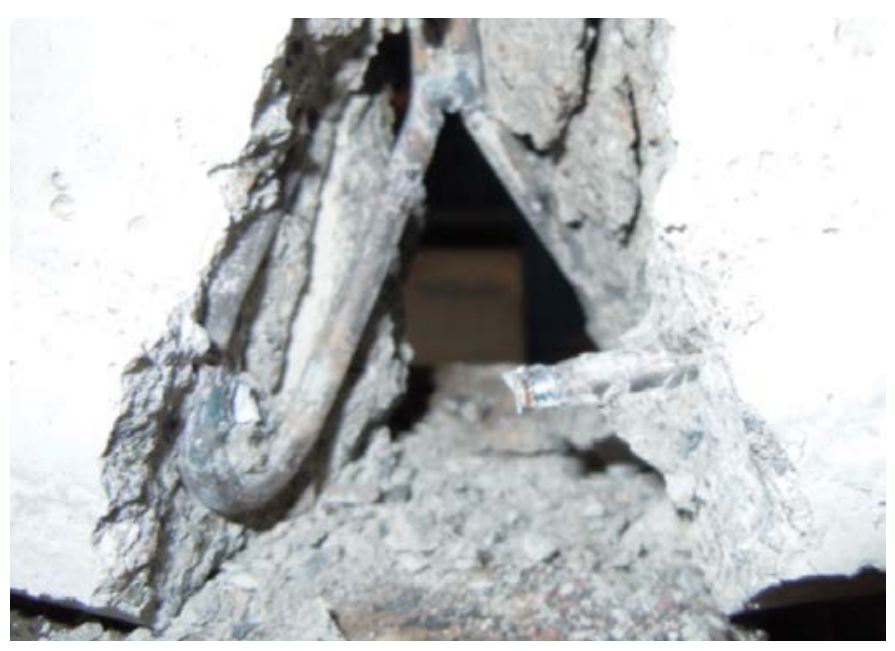

Figura 15. Falla de acero de refuerz en punto de unión soldada

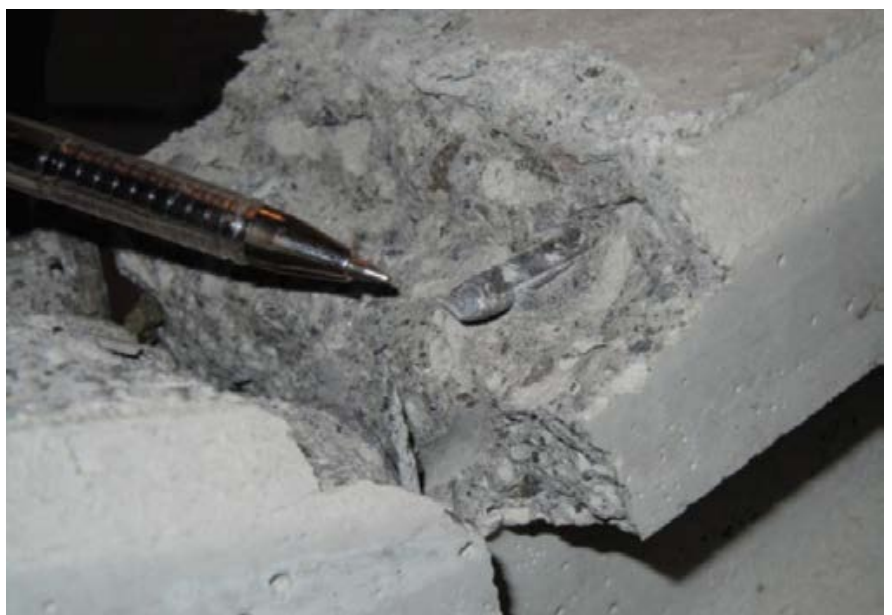

Figura 16. Reducción de diámetro de acero de refuerzo en punto de unión soldada una disminución notable en los momentos obtenidos en relación con el método de voladizo. Estas fallas se producen en el tramo central donde actúa el momento máximo. Para los fabricantes $C$ y $D$ se presentaron fallas cercanas a los puntos de aplicación de la carga.

En las figuras 14, 15 y 16 se muestra el agrietamiento típico en donde se evidencia fallas del acero de refuerzo en puntos donde se realizó una unión soldada.

\section{Análisis de resultados y discusión de los métodos}

De la Tabla 2, se puede observar que aunque las varianzas son estadísticamente iguales, se comprueba que existe un comportamiento atípico de los especímenes del fabricante C. Como se puede observar en la Tabla 3, mediante el método de la capacidad en flexión con carga aplicada en el voladizo, el fabricante $C$ alcanza una resistencia al momento aplicado, superior al promedio de los otros cuatro fabricantes, sin embargo, en la prueba de capacidad en flexión con carga en el tercio medio, el promedio de las resistencias obtenidas del ensayo de sus especímenes, es muy similar al resto de muestras, por lo que los datos de las relaciones entre estas capacidades no tienen un significado físico importante para fines de este estudio, sino que responden a la particularidad constructiva de que existe una sección en el centro de la sección que se encuentra susceptible a falla por fragilidad en la unión soldada cuando se le aplica una carga mediante el método del tercio medio.

En la figura 14 se muestra como el inicio de agrietamiento sucede en uno de los puntos de aplicación de carga. En esta sección el espécimen está sometido tanto a momento máximo como a cortante máximo. Como se muestra en las figuras 15 y 16, esta sección coincide con el punto de se realiza la unión soldada del refuerzo longitudinal con el refuerzo transversal. Dado que el diámetro del refuerzo utilizado es pequeño, la unión soldada genera una alteración importante en el desempeño del refuerzo. Uno de los efectos más importantes es la reducción de la sección transversal de la barra. Al utilizar soldadura de arco para unir piezas de espesor pequeño, la unión se vuelve muy sensible al efecto de la intensidad de la corriente utilizada y el diámetro del electrodo. Existe una alta probabilidad que la penetración que se produce al fundir los tres materiales en la unión (el material de las dos piezas a unir y el material del electrodo) reduzca significativamente el diámetro, disminuyendo localmente su resistencia a la tensión.

Otro efecto que produce el utilizar este tipo de unión soldada está asociado con el calentamiento y enfriamiento 
del material. Cuando se realizan cambios térmicos abruptos en un metal, su estructura cristalina cambia rápidamente, esto quiere decir que los enlaces metálicos se rompen rápidamente cuando se pasa a un estado líquido de manera instantánea. Al enfriarse, la formación de los nuevos enlaces puede generar más dislocaciones dentro de la estructura cristalina que van a dificultar el movimiento de dislocaciones en el momento que se produce la intrusión al rango plástico, como resultado se produce una reducción significativa en la ductilidad de la unión.

El fabricante D, también mostró esta particularidad, pero en proporciones inferiores. En este caso, se comprobó que la particularidad de este fabricante no afecta los datos de manera significativa así que no se considera un valor atípico dentro de la variación normal de los fabricantes. Esta situación demuestra que la relación de los métodos es susceptible a diferencias en la sección transversal de las columnas. Por lo que no se podría determinar una equivalencia entre los métodos si la sección transversal varía o si varía el refuerzo interno a lo largo del espécimen.

De la Tabla 3, se puede observar que las columnas de los fabricantes $\mathrm{B}$ y $\mathrm{E}$, con refuerzo de acero pretensado, presentan desviaciones estándar mayores sobre todo en las fallas del acero. El fenómeno se puede describir por el proceso constructivo, ya que si el control de la tensión aplicada a los torones no es fiscalizada para mantenerse dentro de una banda corta, sino que se dispone de un límite mínimo y una tensión permisible superior relativamente alta, los resultados de la falla del acero, tendrán tanta variación como las tensiones que se le apliquen. Otra eventualidad, que derivaría este fenómeno es una falta de adherencia en la superficie del acero, que es función de la mezcla de concreto utilizada, la calidad de acero utilizado en la manufactura de la sección y los errores en el procedimiento de construcción donde el torón haya tenido contacto con el desmoldante.

En la tabla 4 y Tabla 6 se puede observar que la desviación estándar con los datos del fabricante $\mathrm{C}$, representan aproximadamente un $33 \%$ del promedio, lo que significa para fines experimentales, que las tolerancias de aceptación para la capacidad en flexión de la sección tendrían bandas límite amplias, ante la variación presentada por este fabricante y que se conoce de antemano que existe una particularidad en el método constructivo. Se deduce que los resultados que se presentan en la Tabla 5, son incluyentes para columnas prefabricadas de concreto reforzado, donde el acero y el comportamiento del mismo es homogéneo en toda la sección sobre la longitud del espécimen. Las particularidades que se pueden presentar entre fabricantes que tienen secciones con un comportamiento heterogéneo y su impacto sobre el procedimiento de control de calidad se pueden derivar de los resultados de la Tabla 4 y Tabla 5.

Partiendo de los resultados mostrados en la tabla 5, se puede observar con claridad que aparentemente no existe diferencia significativa entre ambos métodos. Los valores promedio de la relación del momento de agrietamiento y de falla obtenido con el método de carga en voladizo con el momento de agrietamiento obtenido con el método de cargas en los tercios son de 1,02 y 1,03 respectivamente. Inclusive para los valores de momento de agrietamiento, que son definidos mediante la aparición de una grieta con una abertura establecida, la diferencia entre los métodos es menor de $3 \%$.

Es posible realizar un análisis de fuerzas internas para describir la relación que existe entre las fuerzas que se generan en la sección crítica en el método del voladizo (punto de empotramiento) y las que se generan en la sección crítica en el método de cargas en los tercios. Para este análisis, se toma como sección critica para el método de cargas en los tercios, los puntos de aplicación de carga, ya que allí se produce una distribución de fuerzas internas similar a la que sucede en el método del voladizo, donde se tiene un cortante aproximadamente constante y donde el momento varía aproximadamente de forma lineal hasta el máximo que ocurre precisamente en el punto de aplicación de la carga. Esta distribución se presenta desde el apoyo hasta el punto de aplicación de la carga. Como se ha mencionado anteriormente, la sección central está sometida a momento en ausencia de cortante. Para realizar el análisis de fuerzas internas, se desea encontrar una relación entre las cargas necesarias para alcanzar el momento máximo en las secciones críticas para cada metodología de ensayo.

Para efectos de este análisis, dado que los valores de las longitudes de las muestras utilizadas en esta investigación, son de 3,15 a 3,30 m, se utiliza un valor promedio de 3,23 $\mathrm{m}$ para agilizar los cálculos. De esta forma en el caso del método de cargas en los tercios, la distancia entre apoyos puede representarse como $0,97 \mathrm{~L}$.

Despreciando el efecto del peso de los aparejos se pueden igualar las ecuación [1] con la ecuación [2] y obtener la relación entre las cargas para el método de cargas en los tercios comparado con el método en voladizo; el resultado obtenido es el siguiente:

$$
P_{T}=3,14 * P_{V}-0,822 * W_{c}
$$


Donde:

Pt corresponde a la carga máxima para el ensayo de cargas en los tercios

Pv corresponde a la carga máxima para el ensayo en voladizo.

De los resultados se puede demostrar que el valor promedio de la carga Pv es de $270 \mathrm{kgf}$ por lo que el peso promedio Wc representa aproximadamente un $37 \%$ de $\mathrm{Pv}$, considerando esto se puede establecer que

$$
P_{T}=2,84 * P_{V}
$$

Con esta información se puede obtener que el cortante asociado al ensayo de voladizo es básicamente igual a la carga $\mathrm{Pv}$ y que el cortante para el caso de carga en los tercios es de 1,45 Pv.

De los resultados obtenidos se puede decir que este incremento en las fuerzas cortantes que actúan en la sección crítica no afecta el comportamiento de columnas que presentan un refuerzo continuo, sin puntos de concentración de esfuerzo o puntos de fragilidad. En otros casos (fabricante $\mathrm{C}$ ) este fenómeno puede generar una reducción de hasta un $50 \%$ en la capacidad medida en flexión por el método de cargas en los tercios.

Este resultado también indica que la falla de estos elementos en condiciones de refuerzo continuo está regida por flexión y por ende la diferencia en la forma que se apliquen cargas al elemento no afecta en su capacidad.

Con respecto a la diferencia de los métodos en cuanto a su facilidad experimental, el método del voladizo presenta el inconveniente que se debe tener especial control sobre la carga de empotramiento en la base. La carga allí aplicada debe mantenerse constante durante la ejecución del ensayo, esto obliga a que el ensayo deba ser realizado por dos técnicos o por un técnico utilizando un equipo con un servo-control manejado por un computador que regule la aplicación de carga de forma automatizada de manera que el valor sea constante.

Otra ventaja del método de cargas en los tercios, es la facilidad de montaje. En el método de voladizo, es necesario realizar el montaje de manera que luego de empotrar la base en la máquina, el pistón del gato de ensayo, provea un apoyo al espécimen. Esto hace que se deba colocar el espécimen con especial cuidado, demorando la duración del ensayo
Considerando que aparentemente no existe diferencia significativa de los resultados de la capacidad en flexión obtenidos por ambos métodos, y dado que el método de cargas en los tercios ofrece una ventaja técnica en cuanto a la facilidad de montaje, complejidad de equipos requeridos y el nivel técnico requerido en los operarios asignados a la realización del ensayo, este ensayo podría ser utilizado para evaluar la resistencia en flexión de columnas prefabricadas.

\section{Conclusiones}

Para columnas prefabricadas de concreto reforzado con una sección homogénea a través de la longitud del espécimen se puede utilizar el método de cargas en los tercios para determinar su resistencia en flexión.

El tipo de refuerzo no tiene un impacto significativo sobre los resultados entre las pruebas.

Las uniones soldadas en el refuerzo de acero con susceptibilidad a falla por fragilidad, presentan una perturbación en el comportamiento de la sección en el ensayo de carga aplicada en los tercios medios.

La relación del momento de agrietamiento y de falla obtenido con el método de carga en voladizo con el momento de agrietamiento obtenido con el método de cargas en los tercios (excluyendo fabricante C) son de 1,02 y 1,03 respectivamente.

\section{Limitaciones y recomendaciones}

Las diferencias constructivas que presente cada fabricante, hacen que se tenga que analizar todas las particularidades como una perturbación al estudio.

No obstante el análisis de resultados hace referencia a posibles situaciones que se pudieron generar durante la fase constructiva de las columnas, no fue posible realizar una inspección detalla del procedimiento de fabricación, por lo que se recomienda se realice una investigación específica para comprobar las hipótesis sobre el efecto de concentraciones de esfuerzos en la sección transversal de la sección con los métodos de falla estudiados y el efecto que juega el pre-esfuerzo en los torones de acero en la dispersión de los datos de resistencia a la falla de las columnas de este tipo.

El criterio para definición del momento de agrietamiento fue un parámetro tomado de la experiencia tradicional de la ejecución de este tipo de ensayos. Se recomienda realizar un análisis de las curvas de carga desplazamiento para 
establecer criterios específicos para los diferentes métodos y los diferentes tipos de columnas.

\section{Referencias}

1. American Concrete Institute. $\mathrm{ACl}$ 318-08: Requisitos de Reglamento Para Concreto Estructural y Comentario. ACl - Capítulo Costa Rica. Costa Rica, 2008.

2. American Society for Testing and Materials. ASTM C 78M-10: Standard Test Method for Flexural Strength of Concrete (Using Simple Beam with Third-Point Loading). 4 páginas. Estados Unidos.

3. American Society for Testing and Materials. ASTM E 178-08: Standard Practice for Dealing With Outlying Observations.18 páginas. Estados Unidos.

4. American Society for Testing and Materials. ASTM A615M-12 Standard Specification for Deformed and Plain Carbon-Steel Bars for Concrete Reinforcement. 6 páginas. Estados Unidos.

5. American Society for Testing and Materials. ASTM A706M09b Standard Specification for Low-Alloy Steel Deformed and Plain Bars for Concrete Reinforcement. 6 páginas. Estados Unidos.
6. American Society for Testing and Materials. ASTM A82M-07 Standard Specification for Steel Wire, Plain, for Concrete Reinforcement. 4 páginas. Estados Unidos.

7. American Society for Testing and Materials. ASTM A416M-10 Standard Specification for Steel Strand, Uncoated Seven-Wire for Prestressed Concrete. 5 páginas. Estados Unidos.

8. American Society for Testing and Materials. ASTM A421M 10 Standard Specification for Uncoated Stress-Relieved Steel Wire for Prestressed Concrete. 5 páginas. Estados Unidos.

9. American Society for Testing and Materials. ASTM A910M 10 Standard Specification for Uncoated, Weldless, 2- and 3-Wire Steel Strand for Prestressed Concrete. 4 páginas. Estados Unidos.

10. Banco Hipotecario de la Vivienda Detalle de administración del BFV entre 01/01/2011 al 31/12/2011, disponible en www.banhvi. fi.cr/estadisticas.htm

11. Colegio Federado de Ingenieros y Arquitectos. Código Sísmico de Costa Rica, CSCR-2010. Editorial Tecnológica de Costa Rica. Costa Rica, 2010.

12. Walpole, R; Myers, R; Myers, S; Ye, K. Probabilidad y Estadística para Ingenieros, 8va Edición, 816 páginas. Editorial Prentice Hall. México, 2007. 